\title{
Use of Whole Body Vibration as an Exercise Modality for Older Adults with Health Conditions
}

\author{
Wendy Davis \&Janet L. Currie \\ School of International Studies \& Education \\ University of Technology Sydney \\ 15 Broadway \\ Ultimo NSW 2007 \\ Australia
}

\begin{abstract}
The concept of whole body vibration conjures up a range of images, usually related to the health and fitness sphere. It may be an image of a healthy fit Russian astronaut using a vibration technology to counter the deleterious effects of minimal gravity in space, or that of an obese person lying supine on a machine, effortlessly simulating exercise. Today both of these versions of reality are attainable. Technological advances, combined with the race to claim a portion of the global fitness industry equipment market, have made whole body vibration units accessible to a wider proportion of the population. In this paper we highlight the main findings in the research literature regarding the core features and technological elements of whole body vibration. We discuss the major variables informing the evidence-base of whether whole body vibration provides appropriate stimulation to provide efficacious fitness and therapeutic benefits. We analyse whether whole body vibration could be simply considered another fitness 'fad', adding to other unwanted, obsolete fitness machines destined for storage in the shed such as Abtrainers. Finally, research on the use of whole body vibration as a therapy for aging adults and those suffering from diabetes, and chronic obstructive pulmonary or airways disease is reviewed to ascertain if modern, affordable machines provide opportunities for achieving physical improvements.
\end{abstract}

Keywords: Whole body vibration, Health technology, COPD, Health benefits

\section{Introduction}

Whole body vibration (WBV)is achieved when the body is exposed to stimuli that cause the body to shake. Negative effects of chronic exposure to whole body vibration are well documented. Farming, drilling, and construction are amongst the occupations of most concern. International Standards inform safe rates of exposure. Unlike industrial exposure, the type of WBV used in the health and fitness field is typically for short bursts of time with the level of vibration maximized to encourage health benefits.

WBV is an exercise modality accessed by standing on a platform that vibrates (Merriman, Brahler, \& Jackson, 2011).There is a plethora of different models of this technology available on the market, however Figure 1 illustrates various exercises used with a typical platform. Platforms may or may not provide arm and upper body support. The nature of the vibration is key to the effect on the body. The general premise of WBV is that the stimulation provided by the vibration causes positive changes to the body. It is proposed that these changes can be achieved with minimal voluntary muscle activation, and within a fraction of the time required to complete a traditional aerobic or resistance training session.

Figure 1. Typical WBV machine and exercises (Hypervibe, 2015)
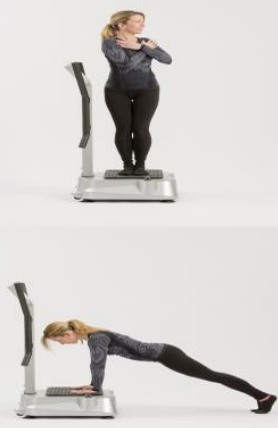
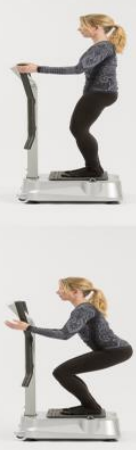
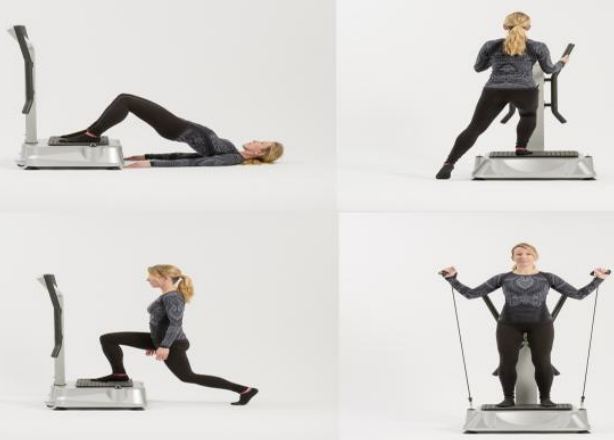
There are two common types of vibration movement. The platform can provide vibration stimulus by moving purely in a vertical plane. That is, the platform rises up and down only. Alternatively, the platform may move around a central pivot point not unlike that of a see-saw commonly found in a children's playground. With the latter mode, the strength of the vibration is dependent on where on the platform the feet are placed. The closer to the centre, the smaller the vibrational impact, and similar the children's seesaw the largest movement is at the furthest lateral point away from the centre.

\section{Key Technical Variables of WBV Machines}

Following a major consultative review, The International Society of Musculoskeletal and Neuronal Interactions published new guidelines in 2010 to help enhance the capacity of the scientific community to more easily understand future research. These guidelines focus on the nature of the WBV stimulation and the terminology used to describe the intervention (Rauch et al., 2010). Whether the vibration is vertical or moves from side to side, there are three key definable criteria at play (Rehn, Nilsson,\& Norgren, 2008; Merriman,\& Jackson 2009; Rauch et al., 2010; Majernik, Dziakova, \&Zivcak, 2013; Blasimann et al., 2014):

1) Time of one cycle. Expanding on the see-saw analogy, starting at the mid-point (equilibrium), the time it takes for one end to go down to the ground, return through equilibrium to its highest point and return to the middle (equilibrium), is the time elapsed for one cycle.

2) Frequency is the number of times this can be done in one second.

3) The displacement is the total size of the movement vertically, from the top to the bottom.

Table 1 outlines defining characteristics of the vibration emitted through WBV platforms. Peak acceleration is the term used to describe the level of vibration. This can be provided utilising the unit of $\mathrm{ms}^{-2}$. The designated acceleration at sea level of the earth's surface is $9.81 \mathrm{~ms}^{-2}$, and this is also known as $1 \mathrm{~g}$. Commonly a ' $\mathrm{g}$ ' is used as a reference point, where $2 \mathrm{~g}$ is twice the peak acceleration of the earth's surface at sea level $\left(2 \times 9.81 \mathrm{~ms}^{-2}\right)$ and $3 \mathrm{~g}$ 's three times the peak acceleration of the earth's surface at sea level. Platforms that provide vibration via the whole platform moving up and down will provide a consistent peak to peak displacement $\mathrm{D}$, whereas $\mathrm{D}$ will vary on other platforms and is dependent on the foot distance from the axis.

Table 1. Characteristics of the vibration provided by WBV platforms (Rauch et al.,2010)

\begin{tabular}{llll}
\hline Term & Definition & Measured in & Denoted by \\
\hline time & $\begin{array}{l}\text { Time taken for one full cycle (that is, the seconds } \\
\text { time it takes to go through one cycle and } \\
\text { return to the starting point - one oscillation) }\end{array}$ & $\mathrm{T}$ \\
\hline frequency & $\begin{array}{l}\text { The number of times the complete cycle Hertz } \\
\text { occurs in one second (the number of } \\
\text { oscillations per second) }\end{array}$ & $\mathrm{Hz}$ \\
\hline displacement & $\begin{array}{l}\text { The size of the movement from the lowest mm } \\
\text { peak (trough) to the highest peak }\end{array}$ & $\begin{array}{l}\mathrm{D} \\
\text { (D=2 x A) }\end{array}$ \\
\hline amplitude & $\begin{array}{l}\text { The size of the movement from the midpoint mm } \\
\text { of equilibrium (the size of the displacement } \\
\text { from equilibrium to peak or from } \\
\text { equilibrium to trough) }\end{array}$ & $\begin{array}{l}\mathrm{A} \\
(\mathrm{A}=\mathrm{D} / 2)\end{array}$ \\
\hline
\end{tabular}

Commercially available WBV machines offer a range of frequency and peak to peak displacements and ultimately exposure to a range of ' $\mathrm{g}$ ' forces. It has been reported that some commercially available WBV machines are capable of delivering vibration therapy in excess of the recommendations in International Standards. If calibrated correctly, WBV machines will accurately deliver vibration to the specifications. Surprisingly, only one study provided details of calibration and drift monitoring on the performance of the machine. Whilst the machine may deliver a defined vibration, an added complexity is the level of vibration delivered is a poor predictor of the vibration effects felt by the body. Muscle stiffness, body weight, footwear, and stance amongst other things can influence the transfer of the vibration to the body.

\section{Safety of WBV Machines and Their Effect on the Body}

Manufacturer guidelines provide a list of conditions thought to be unsuitable for exposure to WBV. Regularly excluded to receiving WBV in clinical studies are patients with acute inflammatory disorders, acute disc herniation, joint fusions with metal implants, recent fractures, acute soft tissue injury, osteoarthritis with vertebral fractures, thrombosis, and malignancy. 
This list is not exhaustive. Sporadic oedema, shin pain and nephrolithiasis have been mentioned as conditions contraindicated for WBV, yet have not been fully investigated through clinical studies nor the adverse events completely attributable to WBV treatment(Robbins et al., 2012).Low grade transient erythema in the lower extremities may occasionally occur from WBV, however is well tolerated and self-resolving (Russo et al., 2003). Studies have shown good tolerance of the exercise modality in the aged, chronically ill, and even by those hospitalised with acute exacerbations of chronic obstructive pulmonary disease (Sa-Caputo et al., 2014; von Stengel et al., 2009). Despite these findings it is recommended that participants be gradually exposed to the vibration stimuli.

While Jones, Gordon and Sealey (2013) found that WBV has the ability to increase muscle power with improvements in strength, dynamic and static balance and general wellbeing, large scale clinical studies of the health or contraindicated effects of WBV are relatively non-existent. Particularly sparse are studies indicating use of appropriate control groups or adequate details of the experimental protocols leaving doubt as to whether stated benefits were purely the result of increased activity as opposed to WBV intervention (Marin \& Rhea 2010; Merriman, Brahler, \& Jackson 2011; Rogan et al., 2011; Chanou et al., 2012). On occasions, studies have also not been clear in stating which type of vibration the participants were exposed to, that is, vertical or side alternating. Observable macro-measurable improvements are unconvincing as many participants with chronic diseases demonstrate highly variable indicators of physical capacity from day to day.

During space expeditions in the 1970s, astronauts experienced reduced muscle mass and strength due to the effects of minimal gravity in space. WBV was used to address this issue. Whilst the exact nature of the effect of WBV on the body is still not fully understood, a number of theories have been reported. One theory proposes that the stimulation to muscle fibres provided by WBV evokes activity of the muscle spindle and ultimately activates the TNV reflex (Merriman, Brahler,\& Jackson, 2011). It is this stretch reflex which initiates muscle contraction, (Christie et al., 2013). With WBV, this contraction happens at a speed and rate that the body is unable to voluntarily replicate. It is this muscle flexion that improves the permeability of myocyte membranes to glucose, causing an insulin-like effect, (Behboudi et al., 2011). WBV involves nearly $100 \%$ of the body's muscles whilst traditional exercise involves only $40-60 \%$ of them at any one time (Behboudi et al., 2011; Bosco et al., 1998).

Perchthaler, Horstman,\& Grau (2013) suggest thatthe way WBV works is through activation of the central nervous system, innervating appropriate muscles in response, which attempt to minimise the soft tissue vibration occurring for protection from injury. Bosco et al. (1998) purport that WBV elicits fast biological adaptation connected to neural potentiation. Vibration also elicits an increase in total peripheral resistance. This is thought to be due to the movement of the walls of the vasculature system the body recognizing a decrease in circulation and compensating by opening the peripheral vasculature system (Button et al., 2007; Robbins et al., 2014). This vasoconstrictor reflex and active local vasodilation compete against one another and enhance gas and nutrient exchange in these areas and to the muscle fibres (Robbins et al., 2012). Studies have also reported a decrease in blood inflammatory markers, such as the receptor of TNF-alpha post-WBV intervention (Christie et al., 2013).

Traditionally, resistance training has been the approach taken to improve muscle power. Power is the maximal muscle strength able to be used in the shortest amount of time. Plyometric training for power development is common in highly conditioned athletes, requiring impeccable technique to minimise injury. Even one dose of WBV in young healthy adults of 4 minute exposure at $15-30 \mathrm{~Hz}$ and $3.5-14 \mathrm{~g}$ showed an array of transient improvements including a significant increase in the isometric extension of the lower extremities, improved body balance and jump height. These effects were not evident 60 minutes post intervention (Torvinen et al., 2002).

Bosco et al. (1998) argued that WBV treatment elicits a biological response similar to that which occurs during and after explosive power training, yet with significantly less risk of injury. Over 10 days participants were exposed to $5 \mathrm{x}$ 90 secs at $26 \mathrm{~Hz}$ at almost $3 \mathrm{~g}$, with a total exposure of 100 minutes. They found t exposure to voluntary muscle contractions including 200 drop jumps from $60 \mathrm{~cm}$ twice a week for 12 months did not attain anywhere near the g force or power increases possible from use of the WBV.

Not only does WBV have an effect on muscle strength, an eight week study on healthy young males showed WBV prevented bone loss from the tibia during bed rest (Rittweger, \& Felsenberg, 2004). Vibration has a positive effect on bone strength. It is thought that when the spongy part of the bone is exposed to vibration, it activates osteocytes to regenerate (Christie et al., 2013). A reduction in circulating cortisol levels and increases in growth hormone reducing bone resorption has also been reported (Elmantaser et al., 2012). Just as traditional load bearing resistance training has the capacity to increase bone density or at least decrease its rate of decline, so does WBV. In a meta-analysis of 15 papers, Rogan et al. (2011) reported varying observable effects noted between side alternating and purely vertical WBV systems, although was not able to conclusively determine the difference. Overall, the neuromuscular responses of the body show that WBV provides the benefits of exercise in less time without fatigue or significant risk of injury. 
The meta-analysis studies that are available tend to reveal predictable positive outcomes from WBV treatment, despite not pinpointing precise details of the most optimal training regimes (Marin \& Rhea, 2010; Merriman, Brahler, \& Jackson 2011). To be effective over the long term it is vital that exercise prescription provides progression of stimuli. Like traditional exercise, clients undergoing WBV need to be continuously exposed to unfamiliar stimulus or overload to result in heightened response to the exercise. Over time, the body becomes comfortable with the applied strain of familiar exercise as a consequence biological change diminishes due to this deficiency of overload. Ideally the aim is to alter the stimulus and combine this with an appropriate increase in stress. This results in the optimum overloading of the physiological system and subsequent chronic adaptations to the exercise occur (Marin \& Rhea, 2010).

\section{Health Benefits of Load-Bearing Exercise for Older Adults}

Load bearing resistance exercise programs are known to have positive effects on muscle strength and is vital for maintaining balance and bone strength - two major issues affecting functional living into older age (Marques, Mota, \& Carvalho, 2012). Increasing age often comes with a range of health and lifestyle issues. Decreased levels of fitness and agility can have substantive negative effects on quality of life contributing to increased morbidity and mortality. TheWorld Health Organisation states falls as being the $20^{\text {th }}$ most common cause of death in the world in 2015 (WHO, 2013). Even minor falls can have a devastating effect on the quality of life of an aging individual, robbing them of confidence and independence (Beaudart et al., 2013).

It is widely accepted that a decrease in bone mass density and subsequent frailty increases the risk of bone fracture as we age. Minimising fragility through maximising bone density as we age is an important health goal for older individuals. In a systematic review of the literature, Merriman and Jackson (2009) analysed 13 WBV studies measuring bone density in the hip, tibia and the lumbar spine. Studies consistently showed improvements in bone density associated with WBV in both the hip and tibia, but not the lumbar spine. Individual studies have however, shown marginal improvements in Bone Mineral Density of the lumbar spine, albeit with less than optimal control mechanisms in place. Similarly, Russo et al.'s (2003) study of post-menopausal women found that tibial cortical bone density remained stable in those exposed to the WBV regime, but significantly declined in the control group. Whilst the therapeutic protocol was not established, it is clear that appropriate exposure to WBV is highly likely to have a positive impact on bone density of the tibia and hip. It is estimated that $30 \%$ of post-menopausal have osteoporosis. Given the mortality of hip fracture is in the order of $20 \%$, there is a real opportunity to use WBV more widely in the community to improve bone density.

There is a level of complexity involved for researchers measuring the effects of WBV. Large variations in balance are frequently noticed by many as they go about their daily routine. Some days are better than others. Generally, however, individuals tend to self-report long term improvements in balance by their ability to consistently dress (socks, shoes and legs of pants), for example with ease, without concern of falling, or without the need to sit.

In terms of the positive impact possible from WBV on health and balance of older people, numerous studies have been able to show that WBV plus physical therapy performs better than physical therapy alone. For example, Kawanabe et al. (2007) found improvements on measures such as improved maximum standing time on one leg and movement velocity, with training of 4 minutes at $12-20 \mathrm{~Hz}$ for once a week for two months. Despite this finding, Rogan et al. (2011) reported on weak to moderate evidence indicating improvement in static and dynamic balance found postintervention in the elderly. An 11-week study carried out by Gomez-Cabello et al. (2012) using the vertical vibration platform failed to show improvements in balance, despite showing other improvements to overall wellbeing.

A 6-month randomised controlled trial studying the effect of WBV concluded that WBV plus squat training is safe and beneficial in improving body balance and muscle power on elderly patients with knee osteoarthritis and/or spondylosis (Osugi et al., 2014). Even a single exposure to $\mathrm{WBV}$ at $6 \mathrm{~Hz}$ with a peak to peak displacement of $10 \mathrm{~mm}(\mathrm{D}=10)$ for ten minutes invoked a statistically significant change in single limb balance for both legs (Jones, Gordon,\& Sealy, 2013).

In researching new ways of reducing the risk of osteoporotic fractures, 151 post-menopausal women were recruited to the Erlangen Longitudinal Vibration Study (ELVIS). Participants were randomly prescribed different yet comprehensive exercise regimes with or without an additional dynamic six minutes of vertical WBV with a peak to peak displacement of $2.4 \mathrm{~mm}(\mathrm{D}=2.4)$ at between $25-35 \mathrm{~Hz}$ for 18 months. In this case no significant difference was noticed between those exposed to WBV and those who were not (von Stengel et al., 2009). This information suggests that the benefits of WBV are most likely to impact those who are not able to or willing to participate in alternative more vigorous exercise. As we age the rate of loss of strength and muscle power increases. The more deconditioned we become, the quicker the decline. Muscle strength decline is known to be much more pronounced in the lower extremities than in the upper extremities (Mester, Kleinoder,\& Yue 2005). 
Traditional resistance training programs will usually have a positive impact, however, not surprisingly, muscle fatigue, soreness and motivational issues are frequently cited as reasons for non-compliance or adherence issues.

Various studies demonstrate a level of improvement in muscle power possible after exposure to WBV. Russo et al. (2003) progressively increased exposure of post-menopausal women to side alternating WBV from $12-28 \mathrm{~Hz}$ and from 3-6 minutes over six months, demonstrating a 5\% improvement in muscle power. Other studies have demonstrated at $15 \%$ improvement in knee extensor dynamic strength over 24 weeks(Merriman, \& Jackson, 2009). Gomez-Cabello et al (2012) exposed 49 non-institutionalised older adults (between 70 and 80 years of age) to 11 weeks of WBV training at $40 \mathrm{~Hz}$ with a displacement of $2 \mathrm{~mm}(\mathrm{D}=2)$ for a total of a little over four hours a (weekly) session, eliciting significant increases in both muscular strength and walking speed.

Evidence suggests that WBV is a suitable activity to improve muscle strength and walking speed and that it may be especially attractive for those who are unable or unwilling to commit to regular traditional exercise regimes. (Christie et al., 2013). The research to date, whilst not able to prescribe specific WBV training protocols, is highly suggestive that WBV is a well -tolerated and beneficial activity for improving balance in the aged population (Kawanabe et al., 2007; Merriman, Brahler, \& Jackson, 2011; Osugi,\& Oguma, 2011).

\section{Benefits of WBV for Patients Experiencing Chronic Obstructive Pulmonary Disease (COPD) or Diabetes}

The global death rate due to COPD is 44 in every 100,000, or the fourth most prevalent cause of mortality in 2015. However, it is projected to increase to the number three cause of death by 2030 (WHO, 2013).COPD is an overarching term encapsulating a number of lung disorders. It causes significant breathing difficulties and is most commonly found in ageing adults. The disease and its treatment regimens can lead to an intolerance of exercise and skeletal muscle function incapacity, independent of the degree of airway obstruction being experienced (Chanou et al., 2012; Greulich et al.,2014; Pleguezuelos et al., 2013).

It is therefore not surprising that WBV has been explored for its potential benefits as an exercise regime for this group. WBV elicited clinically significant improvements in the 6-minute walk test for patients experiencing severe COPD in a randomised controlled trial of outpatients (Pleguezuelos et al., 2013).Greulich et. al. (2014) conducted a randomised clinical trial on patients hospitalised for exacerbations of COPD and found WBV to be a safe exercise option for this acute cohort. When compared with use of physiotherapy alone, WBV therapy induced clinically significant improvements in 6-minute walking tests, increased serum levels of irisin and peroxisome-proliferator-activated receptor-gamma coactivator 1 alpha (PGC1-alpha), and decreased serum interleukin-8 levels, markers of muscle activity(Greulich et al., 2014). The evidence suggested that WBV is likely to produce improvements in function and is a safe and accessible exercise option for sufferers of COPD.

The WHO predicts the global mortality due to Diabetes Mellitus to be 21 in every 100, 000 people, or the number eight cause of death. In 2030,Diabetes Mellitus is expected to be the $5^{\text {th }}$ most prolific cause of death globally (WHO, 2013). Treatment regimens for this heterogenic metabolic disease characterised by an increase in glucose in the blood, usually involve a combination of medication, and attempts at weight loss combining nutrition and physical activity.

Muscle strength and balance can be impaired in patients with diabetes. Patients who are likely to have approximately $15 \%$ reduction in the strength of ankle flexors and ankle extensors. Exercise is prescribed for both prevention and management of the disease. Specific benefits of exercise include improved glycaemic control, lower blood pressure, improved lipid profile and an increase in insulin sensitivity (Baum, Votteler,\& Schiab, 2007; Behbodi et al., 2011; Gordon et al., 2009). Diabetics can also benefit from balance training to help rectify proprioceptive deficits of the foot and ankle which may lead to balance impairment.

Diabetes is a health condition whereby patients often experience a high degree of morbidity and comorbidity. Obesity with large amounts of central body fat occurs frequently in the diabetic population. A group of obese diabetics (with a basal metabolic indicator (BMI) of less than 40) were exposed over a 10-week period to 2 x14 minutes of unloaded static leg and arm WBV exercises per week, at a $40-60 \mathrm{~Hz}$ with a displacement of $4-10 \mathrm{~mm}$. The study reported significant results including decreases in BMI, fat mass, several body part circumferences (Milanese et al., 2013). Sanudo et al. (2013) examined the effect of a 12 week WBV on body composition and leg blood flow in patients with diabetes mellitus Type 2 . The exposure was 12-20 minutes of dynamic and static exercises, at $12-16 \mathrm{~Hz}$ with a peak to peak displacement of $4 \mathrm{~mm}$. The study found a decrease in body fat of $7 \%$, and a decrease in waste circumference by $6 \mathrm{~cm}$ as well as increase leg blood flow velocity. Given the risk of central body fat and the development of insulin resistance and metabolic syndrome, the improvement in adiposity attributable to WBV is significant. Unfortunately, Sanudo et al.'s (2013) study omitted to include a control group performing the same exercises, but without the WBV stimulus. 
Diabetic neuropathy is the most common neuropathy experienced in the populations of industrialised countries. Peripheral small fibre neuropathy affects $1 \%$ of the world's population, and $50 \%$ of the elderly diabetic population (Hong, Barnes,\& Kessler, 2012; Said, 2007). Symptoms can vary; however, patients frequently experience pain, trophic changes in the feet and autonomic disturbances. A case study exploring the use of WBV therapy in the treatment of diabetic peripheral small fibre neuropathy of approximately two years duration found that after four weeks pain level and gait patterns improved significantly (Hong, Barnes,\& Kessler, 2012). The 64 year old received four sets of 3 minute of vibrations (12 minutes per session), at $20 \mathrm{~Hz}, 5$ days a week for four weeks, a total of $4 \mathrm{hrs} \mathrm{WBV}$ for the study period. Similarly, a 6-week case study of a 52 year old diabetic patient who complained of sleep disturbance due to feet tingling and pain in the last 12 months due to neuropathy found WBV helpful in alleviating their symptoms. Muscle strength, balance and flexibility also improved, as well as the waist to hip ratio and BMI. This progressive overload of increasing the time of exposure to $30 \mathrm{~Hz}$ at $4 \mathrm{~mm}$ displacement reduced the pain alleviating sleep disturbances and fear of falling during the day (Yoosefinjad et al., 2012).

An 8-week study with a non-active control group compared walking for 30-60 minutes conducted at $60-70 \%$ of maximum heart rate, with 8-12 minutes of WBV treatment at $30 \mathrm{~Hz}$ and $4 \mathrm{~mm}$ displacement, all three times a week, for Type 2 diabetics. Both the walking group and the WBV group showed similar decreases in fasting glucose at the end of the study period. Results suggested that WBV offers a quicker and more passive, so therefore safer and more accessible, option to aerobic exercise in the management of diabetes mellitus Type 2 or for patients restricted to bed rest (Baum, Votteler, \& Schiab 2007; Rittweger \& Felsenberg, 2004). Overall the literature suggests WBV is a safe and useful tool (when used within parameters) for the diabetic (Baum, Votteler,\& Schiab, 2007; Gordon et al., 2009; Chanou et al., 2009; Sanudo et al., 2013).

\section{Conclusion}

WBV is still often overlooked by health professionals as an exercise modality or considered a novelty approach by most. However, it is recommended that further studies into the efficacy of this technology be conducted. The ease of use, short duration and minimum effort required by users is appealing, particularly for those participants who find traditional exercise difficult or difficult to maintain. Compliance with treatment may be enhanced when participants are introduced to WBV at lower frequencies and duration to allow gradual familiarity to the vibrating sensation prior to exposure to higher levels of stimulation.

Conclusive evidence is yet to be provided, however studies to date strongly suggest that WBV is a safe, viable treatment option for the adult population. It is useful for older adults and those suffering from diabetes or COPD. It is safe, easy to use, well tolerated and with progressive overload regimes produces results comparable to traditional exercise yet in less time and with less effort. There is enough evidence to date for recommending inclusion of WBV as part of a therapeutic treatment protocol for the population groups discussed.

\section{References}

Baum, K., Votteler, T., \&Schiab, J. (2007). Efficiency of vibration exercise for glycaemic control in type 2 diabetes patients.International Journal of Medical Sciences, 4,159-163.

Beaudert, C., Maquet, D., Mannarino, M., Bucknix, F., Demonceau, M., Crielaard, J., Reginster, J., \&Bruyere, O. (2013). Effects of 3 months of short sessions of controlled whole body vibrations on the risk of falls among nursing home residents.BMC Geriatrics, 13, 32.

Behboudi, L., Azarbayjani, M., Aghaalinejad, H., \&Slavati, M. (2011). Effects of aerobic exercise and whole body vibration on glycaemia control in type 2 diabetic males. Asian Journal of Sports Medicine, 2, 83-90.

Blasimann, A., Fleuti, U., Rufener, M., Elfering, A., \&Radlinger, L. (2014).Electromyographic activity of back muscles during stochastic whole body vibration.Journal of Musculoskeletal Neuronal Interaction, 14,311-317.

Bosco, C., Cardinale, M., Tsarpela, O., Colli, R., Tihanyi, J., von Duvillard, S.P., \&Viru, A. (1998). The influence of whole body vibration on jumping performance. Biology of Sport, 15, 157-164.

Button, C., Anderson, N., Bradford, C., Cotter, J.D., \&Ainslie, P.N. (2007). The effect of multidirectional mechanical vibration on peripheral circulation of humans.Clinical \& Physiological Function \&Imaging, 27,211-216.

Chanou, K., Gerodimos, V., Kratrantoau, K., \&Jamurtas, A. (2012). Whole-body vibration and rehabilitation of chronic diseases: A review of the literature. Journal of Sports Science and Medicine, 11,187-200.

Christie, C., Collado, P.S., Marquez, S., Garatachea, N., \&Ceuvas, M.J. (2013).Whole-body vibration training increases physical fitness measures without alteration of inflammatory markers in older adults.European Journal of Sports Science, 14,611-619. 
Elmantaser, M., McMillan, M., Smith, K., Khanna, S., Chantler, D., Panarelli, M., \&Ahmen, S.F. (2012). A comparison on the effect of two types of vibration exercise on the endocrine and musculoskeletal system.Journal of Musculoskeletal \& Neuronal Interaction, 12,144-154.

Greulich, T., Nell, C., Koepke, J., Fechtel, J., Franke, M., Schmeck, B., Haid, D., Apelt, S., Filipovic, S., Kenn, K., Janciauskiene, S., Volgelmeirer, C., \&Koczulla, A. (2014).Benefits of whole body vibration training in patients hospitalised for COPD exacerbations: A randomised clinical trial. BMC Pulmonary Medicine, 14, 60.

Gomez-Cabello, A., Gonzalez-Aguero, A., Ara, I., Casajus, J.A., \&Vicente-Rodriguez, G. (2012).Effects of short-term whole body vibration intervention on physical fitness in elderly people.Maturitas, 74,276-278.

Gordon, B.A., Benson, A.C., Bird, S.R., \&Fraser, S.F. (2009). Resistance training improves metabolic health in type 2 diabetes: A systematic review. Diabetes Research and Clinical Practice, 83,157-175.

Hong, J., Barnes, M., \&Kessler, N. (2012). Case Study: Use of vibration therapy in the treatment of diabetic peripheral small fiber neuropathy. Journal of Bodywork \& Movement Therapies, 17, 235-238.

Hypervibe. (2015). Full body whole body vibration routine - Intermediate level. Hypervibe. Whole Body Vibration.Retrieved from https://www.hypervibe.com/au/blog/full-body-whole-body-vibration-routineintermediate-level/

Jones, A., Gordon, S., \&Sealey, R. (2013). The effect of single session whole body vibration on mobility, lower limb joint position sense and balance of community dwelling older adults. Internet Journal of Allied Health Sciences and Practice, 11, 1-6.

Kawanabe, K., Kawashima, A., Sashimoto, I., Takeda, T., Sato, Y, \&Iwamota, J. (2007). The effect of whole-body vibration exercise and muscle strengthening, balance, and walking exercises on walking ability in the elderly. Keio Journal of Medicine, 56, 28-33.

Majernik, J., Dziakova, M., \&Zivcak, J. (2013).Utilization of Whole Body Vibration in Therapy of Patients with Neurological Disorders, Presented at the International Workshop on Digital and Biomedical Technologies, $1^{\text {st }}$ May, 2013, Zilina, Slovakia.

Marin, P.J., \&Rhea, M.R. (2010). Effects of vibration training on muscle power: a meta-analysis.Journal of Strength and Conditioning Research, 23,871-878.

Marques,E.A., Mota, J., \&Carvalho, J. (2012). Exercise effects on bone mineral density in older adults: A metaanalysis of randomized controlled trials. Age, 34,1493-1515.

Merriman, H., \&Jackson, K. (2009). The Effects of Whole-Body Vibration Training in Aging Adults: A systemic review. Journal of Geriatric Physical Therapy,32, 134-144.

Merriman, H.L., Brahler, C.J., \&Jackson, K. (2011). Systematically controlling for the influence of age, sex, hertz and time post-whole-body vibration exposure on four measures of physical performance in community-dwelling older adults: a randomized cross-over study. Current Gerontology and Geriatrics Research, Article ID 747094. http://dx.doi.org/10.1155/2011/747094

Mester, J., Kleinoder, H., \&Yue, Z. (2006). Vibration training: benefits and risks.Journal ofBiomechanics, 39,10561065.

Milanese, C., Piscitelli, F., Zenti, M.G., Moghetti, P., Sandri, M., \&Zancanaro, C. (2013). Ten-week whole-body vibration training improves body composition and muscle strength in obese women', International Journal of Medical Sciences, 10,307-311.

Osugi, T., Iwamoto, J., Yamazaki, M., \&Takakuwa, M. (2014). Effect of a combination of whole body vibration exercise and squat training on body balance, muscle power, and walking ability in the elderly. Therapeutics and Clinical Risk Management, 10, 131-138.

Perchthaler, D., Horstmann, T., \&Grau, S. (2013). Variations in neuromuscular activity of thigh muscles during wholebody vibration in consideration of different biomechanical variables. Journal of Sports Science and Medicine, 12, 439-446.

Plegeuzuelos, E., Perez, M.E., Guirao, L., Samitier, B., Costea, M., Ortega, P., Gonzales, M.V., Delcarmin, V.A., Ovejero, L., Moreno, E., \&Miravitlles, M. (2013). Effects of whole body vibration training in patients with severe chronic obstructive pulmonary disease. Respirology, 18, 1028-1034.

Rauch, F., Sievanen, H., Boonen, S., Cardinale, M., Degens, H., Felsberg, D., Roth, J., Schoenau, E., Verschueren, S., \&Rittweger, J. (2010). Reporting whole-body vibration intervention studies: Recommendations of the International Society of Musculoskeletal and Neuronal Interactions. Journal of Musculoskeletal Neuronal Interactions, 10, 193-198.

Rehn, B., Nillson, P., \&Norgren, M. 2008, Effects of whole-body vibration exercise on human bone density Systematic review. Physical Therapy Reviews, 13, 427-432. 
Rittweger, J., \&Felsenberg, D. (2004). Resistive vibration exercise prevents bone loss during 8 weeks of strict bed rest in healthy male subjects: Results from the Berlin BedRest (BBR) Study, Poster 1145. Paper presented at the 26th Annual Meeting of the American Society for Bone and Mineral Research, USA, 2004.

Robbins, D., Yoganathan, P., \&Goss-Sampson, M. (2014). The influence of whole body vibration on the central and peripheral cardiovascular system, Clinical Physiology \&Functional Imaging, 34,364-369.

Robbins, D., Zeinstra, E., Jimenez, A., \&Goss-Sampson, M. (2012). Does whole body vibration have clinically significant neurophysiological and neurovascular implications? International Journal of Prevention and Treatment, 1, 18-26.

Rogan, S., Hilfiker, R., Herren, K., Radinger, L., \&de Bruin, E. (2011). Effects of whole-body vibration no postural control in elderly: a systemic review and meta-analysis.BMC Geriatrics, 11, 72.

Russo, C. R., Lauretani, F., Bandinelli, S., Bartali, B., Cavazzini, C., Guralnik, J. M., Ferrucci, L. (2003). Highfrequency vibration training increases muscle power in postmenopausal women. Archives of Physical \& Medical Rehabilitation, 84, 1854-1857.

Sa-Caputo, D., Ronikeili-Costa, P., Carvalho-Lima, R.P., Bernardo, L.C., Bravo-Monterio, M.O, Costo, R., MoraesSilvan, J, Paiva, D.N., Machado, C.B., Matilla-Giehl, P.M., Arnobio, A., Merin, P.D., \&Bernado-Filho, M,B. (2014). Whole body vibration exercises and the improvement of the flexibility in patients with metabolic syndrome. Rehabilitation Research and Practice, Article ID 628518.

Said, G. (2007). Diabetic neuropathy- a review. Natural Clinical Practice \&Neurology, 3,331-40.doi: 10.1038/ncpneuro0504

Sanudo, B., Alfonso-Rosa, R., del Pozo-Cruz, B., del Pozo-Cruz, J., Galiano, G., \&Figueroa, A. (2013). Whole body vibration training improves leg blood flow and adiposity in patients with type 2 diabetes mellitus.European Journal of AppliedPhysiology, 113,2245-2252.

Torvinen, S., Kannus, P., Sievanen, H., Jarvinen, T.A.H., Pasanen, M., Kontulainen, S., Jarvinen, T.L.N., Jarvinen, M., Oja, P., \&Vuori, I. (2001). Effect of a vibration exposure on muscular performance and body balance. Randomised cross-over study. Clinical Physiology and Functional Imaging, 22,145-152.

von Stengel, S., Kemmler., L., Engelke, K., \&Kalender, W.A. (2011).Effects of whole body vibration on bone mineral density and falls - Result of the randomized controlled ELVIS study with postmenopausal women. OsteoporosisInternational, 22,317-325.

World Health Organisation (WHO).(2013).Disease burden and mortality estimates. Retrieved from https://www.who.int/healthinfo/global_burden_disease/estimates/en/index1.html

Yoosefinejad, A.K., Shadmehr, A., Olyaei, G., Talegian, S., Bagheri, H., \&Mohajeri-Tehrani, M. (2012). Effects of whole-body vibration on a diabetic type 2 patient with peripheral neuropathy. Health Science Journal, 6, 576583. 